\title{
User Profiling for Coordinated and Integrated Care
}

\author{
Reem Kayyali ${ }^{1}$, Shereen Nabhani-Gebara ${ }^{1,}$ Iman Hesso ${ }^{1}$, Roshan Siva ${ }^{2}$, Evangelos Kaimakamis ${ }^{3}$, \\ Anouk W. Vaes ${ }^{4}$, Martijn A. Spruit ${ }^{4}$, John Chang ${ }^{2}$, Richard Costello ${ }^{5}$, Nada Philip ${ }^{1}$, Member, IEEE, \\ Barbara Pierscionek ${ }^{1}$ and Nicos Maglaveras ${ }^{6}$, Sr Member, IEEE.
}

\begin{abstract}
Integrated care has been increasingly advocated as an approach to promote better coordination of services and quality of care at different levels. In this study, views were elicited from different users of the healthcare system (patients, informal carers and healthcare professionals) in four European countries. The views pertained to current states of care and the role that remote patient monitoring and telehealth in general can play to facilitate effective implementation of integrated care. Overall, services were perceived to be fragmented at different levels ranging from personal to system fragmentation. Approaches such as telehealth, remote patient monitoring and having specialised urgent clinics in primary care were positively perceived by users as possible solutions for tackling fragmented care and for promoting better integration of services. ${ }^{1}$
\end{abstract}

\section{INTRODUCTION}

The definition of integrated care (IC) has varied widely with more than 175 definitions available in the literature [1-4]. Many synonyms have been used to describe IC such as coordinated care, managed care, disease management and care management [2]. Some authors defined IC as an organisational process for achieving continuous care according to patients' holistic needs and views [5]. Others defined IC as the coordination of the care delivery system, involving multiple interventions targeting patients, healthcare professionals (HCPs) and organisations [2,6].

IC is a complex and multifaceted intervention that can occur in different types and at different levels [7]. Types of integration involve: service integration, professional

\footnotetext{
${ }^{\mathbf{1}}$ This research has been partially funded from FP-ICT programme under grant agreement no. 611223 - WELCOME

${ }^{1}$ Faculty of Science, Engineering and Computing, Kingston University, Kingston-Upon-Thames, United Kingdom, KT1 2EE;

${ }^{2}$ Chest Clinic and Research and Development, Croydon University Hospital, Croydon, United Kingdom;

${ }^{3}$ Pulmonary Clinic, General Hospital G.P. Papanikolaou, Thessaloniki, Greece;

${ }^{4}$ Dept. of Research and Education, CIRO+, Centre of expertise for chronic organ failure, Horn, Netherlands;

${ }^{5}$ RCSI Education \& Research Centre, Beaumont Hospital, Dublin, Ireland.

${ }^{6}$ Medical school, Aristotle University of Thessaloniki, Thessaloniki 54124, Greece and the Institute of Applied Biosciences, Center for Research and Technology Hellas.
}

integration, functional integration, organisational integration and system integration. IC can occur at the following levels: at the macro level (system), meso level (organisational, professional) and micro levels (service and personal) [7,8]. The polymorphous nature of the concept [9] renders it difficult to find a single model that can suit all contexts, settings and circumstances [4,7]. Although key lessons can be learned from different successful IC programmes, transferring such experiences might not be successful between countries [4,7]. In essence, IC can be best understood as an approach that has the potential to improve patient care through better coordination [4].

In fact, the concept of IC has been introduced to healthcare (HC) systems to improve coordination, continuity and quality of care $(\mathrm{QoC})[2,4]$. This is becoming increasingly important, given the economic burden of chronic conditions [6], their increased prevalence with the expected increase in the ageing population $[7,10]$ and the complex nature of $\mathrm{HC}$ systems [1]. The management of such chronic conditions necessitates the presence of multifaceted and multiinstitutional levels of care. A review of systematic reviews highlighted the positive effect of IC programmes on QoC [2]. In one study, the implementation of IC intervention for chronic obstructive pulmonary disease (COPD) patients recently discharged from hospital after suffering from exacerbations was associated with a lower number of hospitalisations $(\mathrm{p}=0.02)$, lower re-admission rates $(\mathrm{p}=$ 0.033 ) and an increase in the percentage of patients without admission $(p=0.03)$ in comparison to usual care after 12 months follow-up [11].

Patients and carers perceive IC as a flexible system that addresses their needs by granting them access to appropriate $\mathrm{HC}$ and social care services, involving them in care decisions and optimising follow-up, communication and information provision by HCPs who are responsible for their care while at the same time facilitating the coordination and cooperation between all HCPs involved in their care $[12,13,14]$. For HCPs, IC is perceived as a streamlined process of care that provides clarity and structure to the care delivered [15]. According to HCPs, successful implementation of IC requires: multidisciplinary communication, commitment and support within the 
organisation in the planning and delivery of care, in addition to service user (patient) involvement [16].

Functional integration is an important type of IC because it supports all the other types of integration through linking financial, management and information systems across the health system [8]. The use of modern information and communication technologies (ICT) can be considered as an aspect of functional integration [7], hence the increased need for approaches that employ new technologies such as remote patient monitoring (RPM) and telehealth (TH). RPM stands for a variety of technologies that are used by patients outside the clinical setting for the monitoring of their health conditions, examples of such monitoring devices include: glucometers, blood pressure monitors and weight scales [17].

$\mathrm{TH}$, is a more comprehensive concept, which can be defined as a technology based intervention that allows the remote exchange of data between the HCP and the patient outside the clinical environment to assist in the management and diagnosis of health conditions [18,19]. It involves the use of technological developments to allow such communication. $\mathrm{TH}$ has been seen as a technological innovation that can promote the integration of health and social care [20].

The aim of this study is to identify challenges of the current HC systems (current state of care) in Europe for patients with chronic conditions namely COPD and associated comorbidities and elicit the role that RPM and TH in general can play to facilitate IC implementation.

\section{METHOD}

This is a qualitative study that forms a part of the user requirement evaluation of the EU WELCOME project (http://www.welcome-project.eu) [21]. This part used focus group discussions (FGDs) and semi-structured interviews to provide a detailed account on the perceptions of different stakeholders including patients, informal carers and HCPs on $\mathrm{TH}$ in general and the WELCOME system in particular. In this paper the perceived challenges within the current $\mathrm{HC}$ systems in Europe and the possible solutions proposed as part of this qualitative study will be outlined. The second phase consisted of follow-up semi-structured interviews with HCPs to elicit additional views about the role of $\mathrm{TH}$ in general in IC.

In the first stage of the project evaluation, 8 FGDs were conducted with 32 patients and 23 informal carers (family members) from four European countries: UK, Ireland, Netherlands, and Greece. In addition 23 semi-structured interviews were conducted with $23 \mathrm{HCPs}$ from the 4 European countries. More details on data collection for this phase are described elsewhere [21]. The second/ follow-up stage was done in England where 36 HCPs were interviewed to elicit additional views about the role that TH can play in IC. The interview schedule for this phase included two sections: TH experience and awareness and perceptions about IC and how it could be facilitated by TH. The followup (second phase) interviews with HCPs lasted approximately 1 hour and were carried out between January and March 2015.

All FGDs and interviews were led by a moderator and were audio-recorded, transcribed and translated to English. All transcripts were thematically analyzed.

\section{RESULTS}

\section{A. Fragmented care as an issue in the current healthcare systems}

Patients' perceptions: The issue of fragmented care and lack of communication between different HCPs involved in patient care was raised as a main challenge in the current $\mathrm{HC}$ system by patients in the four countries. Dutch patients highlighted the differences in pulmonary care between primary and secondary/tertiary care settings, stating that the process prior to referral to a pulmonologist was not adequate as referrals to pulmonologists are unduly late with general practitioners (GPs) not being able to tackle some of the COPD-related problems independently. The majority of patients therefore contact the pulmonologist or pulmonary nurse directly if they need any help. The pulmonary nurse was reported as a;

\section{'good intermediary between patient and pulmonologist'.}

A major reason for concern regarding the communication process between HCPs was reported by an Irish patient having COPD and diabetes who described having to stay for 17 hours without medication before undergoing a procedure. She complained that this situation made her feel that she has to watch over her own medication schedule, despite being unwell:

'they said that they should have made a note that I was diabetic. Now, I told them that morning when I went there, [...] it was 5:30 in the morning. I told them and they wrote out the thing that I was diabetic. And they said they didn't see the note. And that's why I was left so long. But they assured me that it wouldn't happen again. But it was too late', and I couldn't believe. I actually complained to the hospital. They put me at risk. I'm the one who takes the medication to keep my diabetes controlled but I couldn't do it while I was in there'.

Informal carers' perceptions: Similar to patients, carers highlighted that HC services are fragmented. In UK, one carer said: 
“We see a different GP each time.”

Moreover, carers indicated that lack of communication between HCPs makes arranging medicines very hard causing delays in getting the medications. Patients feel they have to be assertive to get what they need. The carers in the UK cohort appreciated the respiratory HOT clinic at the Croydon University Hospital, which is a primary health clinic run by a multidisciplinary team from secondary care including respiratory consultant, nurse and physiotherapist. The clinic provides rapid access to help patients with chronic respiratory conditions who are at risk of hospitalisation to avoid hospital admission.

HCPs' perceptions: Fragmentation of communication between different $\mathrm{HC}$ providers for patients suffering from COPD and co-morbidities has been predominantly identified as an issue reflecting fragmented care in the current $\mathrm{HC}$ systems in the four countries. HCPs highlighted different levels of fragmentation: between the different HCPs involved in the care of a patient; between the HC teams in primary care and secondary care; and between health and social care.

"It's not just what I can provide for them as a healthcare professional. It's very much that kind of social care as well and they don't get as much as they should."

"The difficulties...... are the presence of multiple comorbidities which make them very difficult to manage."

"For COPD with diabetes ---- the issues is how to involve the community respiratory team here or the hospital respiratory team for maximising discharge and help reducing further admissions. ",

B. Acceptance of remote home monitoring and sharing of monitoring results as a solution for integrating care

Patients' perceptions: Even though the participating patients had limited daily interaction with technology (limited computer and smartphone use), they were receptive to the idea of TH for home monitoring and integration of care. The majority of patients advocated the idea of self-monitoring and showed their acceptance to share all their monitoring results and parameters with all HCPs involved in their care. Moreover, patients would like to have access to their own measurements to be reassured that they are fine, and they would like their carers to have similar access. However, the Dutch cohort of patients preferred to share monitoring results with HCPs only and were not willing to share this with their carers to avoid additional burdens.

Informal carers' perceptions: Overall, carers had limited interaction with technology on a daily basis but they were receptive to the concept of $\mathrm{TH}$ without due concern. All carers agreed that they would like access to the parameters measured by patients at their leisure. Carers are happy to act upon any recommendations provided such as taking antibiotics, steroids etc. Like patients, carers also like these monitoring results to be shared with HCPs involved in the care of the patient.

HCPs 'perceptions: According to HCPs, results monitored should be available to all HCPs responsible for patient care. All HCPs agreed that everyone involved in patient care should have access to all their monitoring results and that carers should be sent patients' monitoring results as well. They also ought to be alerted if the patient needs to make an action.

\section{Telehealth as a solution for integrating care}

What was obvious from the follow-up interviews is that both concepts: IC and TH were perceived as complementary and facilitators to each other. TH implementation into normal care pathways can act as a tool for integrating secondary and primary care. Some HCPs understand that the main benefit of TH integrated care is the extended capacity that allows many HCPs to discuss clinical issues together regarding patient care. This can occur without the HCP travelling to assess a patient, which consequently saves time and money for the HCP and the patient.

"Telehealth can help facilitate integrated care but the integrated care and telehealth have to occur at the same time and telehealth should be seen as the means by which integrated care will happen."

\section{DISCUSSION AND CONCLUSION}

This paper provides detailed user perceptions about the current fragmented care in four European countries and proposes solutions for tackling this problem. Our results showed different levels of fragmented care perceived by users: at micro level depicted by lack of continuity of care, at meso level between health and social care, between the different HCPs providing care for the same patient with different co-morbidities; and at macro level between primary and secondary care. The problem of services fragmentation in the health systems has been recently highlighted across many European countries as a main barrier to continuity of care and provision of IC for patients with complex and chronic health needs [22]. The report also highlighted the problem of fragmentation between social and health services in several European countries. In England, even though health and social care are under the responsibility of the Department of Health, yet they operate under different constitutional sectors. Dissatisfaction with the current fragmentation of services in England, more specifically the capital has been highlighted by service users, patients and carers [10] similarly to our study. The same problem was 
highlighted in the USA, where the health system also suffers from fragmentation of services resulting in unsustainable cost increases, poor quality, and inequality [23].

This study outlined some solutions to address the issue of fragmented care.

The respiratory HOT clinic: The UK experience exhibited in the respiratory HOT clinic was greatly appreciated by both the patients and the carers. It enables professional integration through the presence of the multidisciplinary team. The service provides the opportunity for the patient to see a respiratory specialist from secondary care in the primary care setting, thus promoting system integration between primary and secondary care. It also ensures continuity of care when seeing the same specialist each time thus promoting service integration at micro level. The Dutch patients in this study were more satisfied with the care received by the pulmonologist in the secondary care clinic and choose them to be the first point of contact for any help instead of the GP.

Remote patient monitoring: Majority of patients in this study were willing to share their monitoring results with all HCPs involved in their care. It is important to note that patients felt that their carers should have access to their data. This in return promotes organisational integration between health and social care since carers operate under social care and there is professional integration between the multiple HCPs at meso level.

Telehealth: This study provides an insight into the TH role in integrating care. Patients showed a positive attitude and willingness to engage with the concept of TH. Similarly, HCPs and carers were very receptive to the concept and were happy to engage in it constructively. It was evident from the follow-up interviews that all HCPs supported the integration of all $\mathrm{HC}$ services under one umbrella. The datasharing nature associated with $\mathrm{TH}$ was seen as an important concept that could lead to the effective implementation of IC. In one recent research, TH has been suggested as a solution to optimise the care process by patients and carers [12]. ICT was highlighted as an important enabler for IC in the literature [7]. TH use will allow a form of virtual consultation for the patient and accessibility of data to carers and multiple HCPs, thus promoting different types and levels of integration; functional, organisational and professional integration at meso level and system integration at macro level.

In summary, the current care was identified by different $\mathrm{HC}$ system users to be fragmented at different levels. Tackling this problem should be considered at different levels to promote effective care integration that puts the patients' needs at the heart of the health system. Approaches such as RPM, TH and specialised urgent clinics were found to act as enablers of IC. However, the different contexts, post implications and resources for IC implementation across the different health systems need to be taken into consideration.

\section{REFERENCES}

[1] Kodner DL, Spreeuwenberg C. Integrated care: meaning, logic, applications, and implications--a discussion paper. Int J Integr Care 2002;2:e12.

[2] Ouwens M, Wollersheim H, Hermens R, Hulscher M, Grol R. Integrated care programmes for chronically ill patients: a review of systematic reviews. Int J Qual Health Care 2005 Apr;17(2):141-146.

[3] Armitage GD, Suter E, Oelke ND, Adair CE. Health systems integration: state of the evidence. Int J Integr Care 2009 Jun 17;9:e82.

[4] Shaw S, Rosen R, Rumbold B. What is integrated care. An overview of integrated care in the NHS.London: The Nuffield Trust 2011.

[5] Mur-Veeman I, Hardy B, Steenbergen M, Wistow G. Development of integrated care in England and the Netherlands: managing across public-private boundaries. Health Policy 2003;65(3):227-241.

[6] Lemmens KM, Nieboer AP, Huijsman R. A systematic review of integrated use of disease-management interventions in asthma and COPD. Respir Med 2009;103(5):670-691.

[7] Goodwin N, Dixon A, Anderson G, Wodchis W. Providing integrated care for older people with complex needs: lessons from seven international case studies. London: The King's Fund 2014.

[8] Valentijn PP, Schepman SM, Opheij W, Bruijnzeels MA. Understanding integrated care: a comprehensive conceptual framework based on the integrative functions of primary care. International Journal of Integrated Care 2013;13.

[9] Kodner DL. All together now: a conceptual exploration of integrated care. Healthc Q 2009;13 Spec No:6-15.

[10] London Health and Care Integration Collaborative. Integrated Care in London: Case for Change. Available at:

https://khub.net/c/document library/get file?uuid=f44f18b4-dc3a-4bbb-bfe91bd592daa6c1 \&groupId $=6088600$.

[11] Casas A, Troosters T, Garcia-Aymerich J, Roca J, Hernandez C, Alonso A, et al. Integrated care prevents hospitalisations for exacerbations in COPD patients. Eur Respir J 2006 Jul; 28(1):123-130.

[12] Wodsku et al. A qualitative study of integrated care from the perspectives of patients with chronic obstructive pulmonary disease and their relatives. BMC Health Services Research 2014, 14:471.

[13] National voices. Integrated care: what do patients, service users and carers want? Available at:

http://www.nationalvoices.org.uk/sites/www.nationalvoices.org.uk/files/what patients want from integration national voices paper.pdf

[14] Berendsen et al. Transition of care: experiences and preferences o patients across the primary/secondary interface- a qualitative study. BMC Health Services Research 2009, 9:62.

[15] Sleeman et al. 'It does not do the care for you': a qualitative study of health care professionals' perceptions of the benefits and harms of integrated care pathways for end of life care. BMJ Open 2015; 5:e008242.

[16] Hogan C, Barry M, Burke M, Joyce P. Healthcare professionals' experiences of the implementation of integrated care pathways. IJHCQA, 2009; 24(5): 334-347.

[17] Technologies To Help Older Adults Maintain Independence: Advancing Technology Adoption. 2009; Available at:

https://www.google.co.uk/?gfe_rd=cr\&ei=jZQqVoizL7Pj8weAq5H4Dg\&gws_rd=ssl\# $\mathrm{q}=$ Technologies +to + Help+ Older + Adults + Maintain + Independence + :Advancing + Techn ology+Adoption.,+ 2015.

[18] Sanders C, Rogers A, Bowen R, Bower P, Hirani S, Cartwright M, et al. Exploring barriers to participation and adoption of telehealth and telecare within the Whole System Demonstrator trial: a qualitative study. BMC Health Services Research 2012, 12:220

[19] Taylor J, Coates E, Brewster L, Mountain G, Wessels B, Hawley MS. Examining the use of telehealth in community nursing: identifying the factors affecting frontline staff acceptance and telehealth adoption. J Adv Nurs 2015 FEB 2015;71(2):326-337. [20] Goodwin N. Will telehealth and telecare thrive or perish in a cold financial climate? Journal of Care Services Management 2009;3(2):116-118.

[21] WELCOME project: What do stakeholders want? In depth analysis of COPD patients, carers and healthcare professional views. Wireless Mobile Communication and Healthcare (Mobihealth), 2014 EAI 4th International Conference on: IEEE; 2014. [22] Nolte E, Knai C, Saltman RB. Assessing chronic disease management in European health systems. 2014.

[23] Stange KC. The problem of fragmentation and the need for integrative solutions. Ann Fam Med 2009 Mar-Apr;7(2):100-103. 